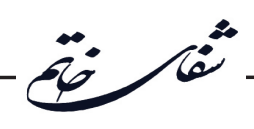

\title{
Effect of Lidocaine on Patient Satisfaction in Spinal Anesthesia
}

\section{Mohammad Fathi ${ }^{1}$, Nilofar Massoudi2 ${ }^{*}$, Ali Dabbagh ${ }^{2}$}

${ }^{1}$ Department of Anes thesiology, Critical Care Quality Improvement Research Center, Shahid Beheshti University of Medical Sciences, Tehran, Iran

${ }^{2}$ Department of Anes thesiology, Anes thesiology Research Center, Shahid Beheshti University of Medical Sciences, Tehran, Iran

\section{Article Info:}

\section{ABSTRACT}

Introduction: One of the most important challenges facing the anesthesiologist before performing painful procedures, such as spinal anesthesia, is the anxiety and fear of patients due to the pain of needle entry into the skin and the consequent lack of cooperation. This study aimed to evaluate the effect of $10 \%$ lidocaine spray on patient satisfaction in spinal anesthesia. Material and methods: In this double-blind clinical trial, patients were referred to a general surgery ward for spinal anesthesia. Patients were randomly divided into two groups. Patients in the first group (lidocaine group) received 10\% lidocaine as anesthesia and patients in the second group received an equal volume of normal saline. Then, based on the Visual Analogue Scale, patients were asked about the extent of pain during needle insertion into the skin and the degree of patient satisfaction was noted. Results: Our data revealed a significant reduction of pain and improvement of fear in patients who received the local application of lidocaine. Conclusion: There was a significant relationship between analgesia, satisfaction, lack of cooperation, and anxiety at the time of needle insertion.

\section{Key words:}

1. Patients

2. Anesthesia, Spinal

3. Personal Satisfaction

*Corresponding Author: Nilofar Massoudi

E-mail: nilomassoudi@gmail.com 
تأثير ليدوكائين بر رضايتمندى بيمار در بيهوشى اسياينال

\section{محمد فتحى'، نيلوفر مسعودى זّبّ، على دباغ'}

اتروه بيهوشى، مركز تحقيقات بهبود كيفيت بحرانى، دانشعاه علوم يزشكى شهيد بهشتى، تهران، ايران

rكَروه بيهوشى، مركز تحقيقات بيهوشى، دانشَاه علوم يزشكى شهيد بهشتى، تهران، ايران

\section{اطلاعات مقاله:}

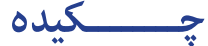

مقدمه: يكى از مهرمترين خالشهاى متخصص بيهوشى قبل از انجام يروسيجرهاى دردناك مثل اسياينال

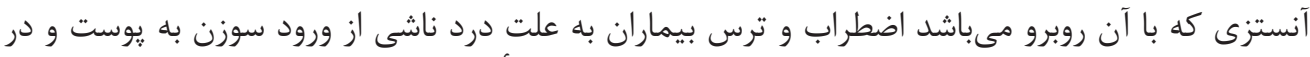

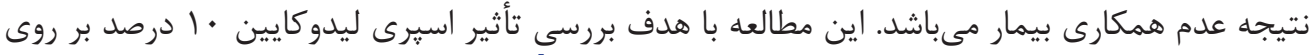

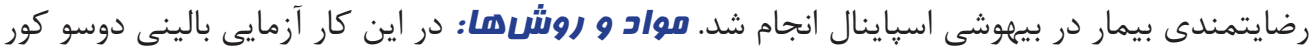

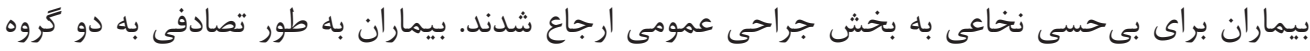

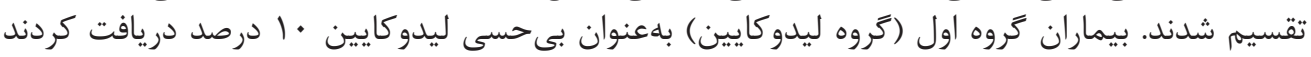

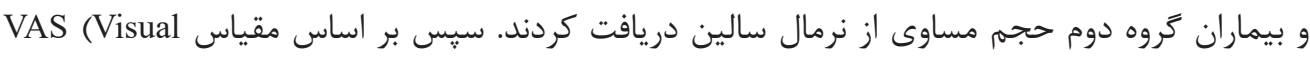
(Analogue Scale

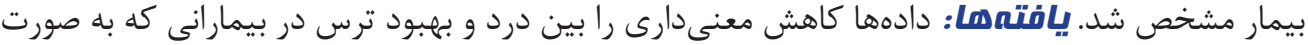

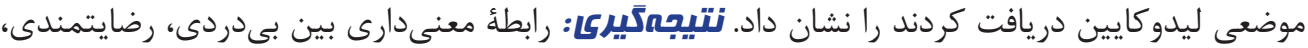

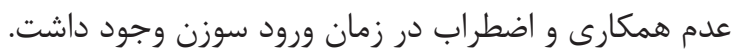

" نويسنده مسئول: نيلوفر مسعودى آدرس الكترونيكى: nilomassoudi@gmail.com 


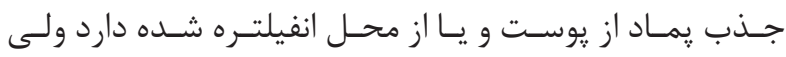

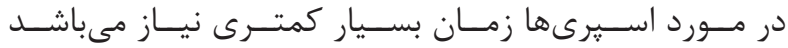

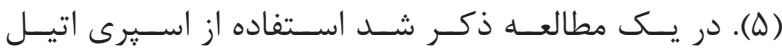

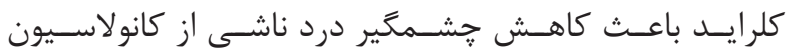

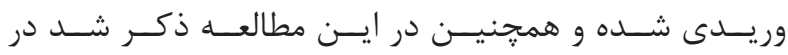

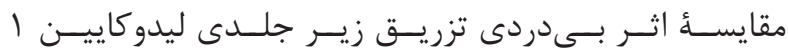

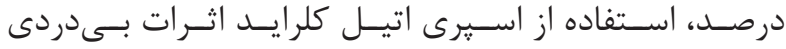

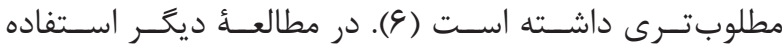

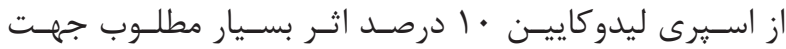

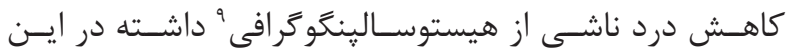

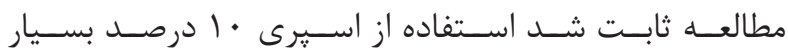

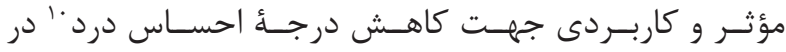

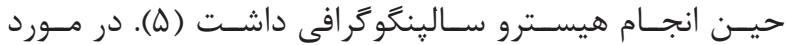

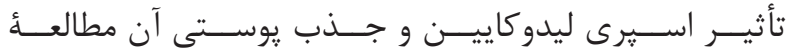

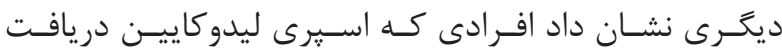

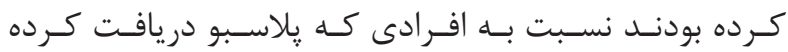

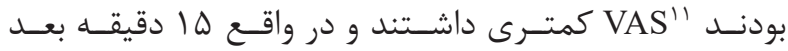

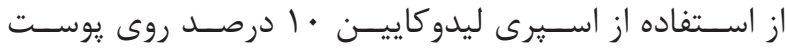

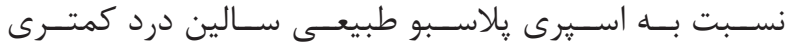

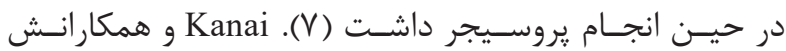

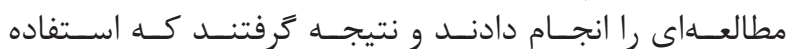

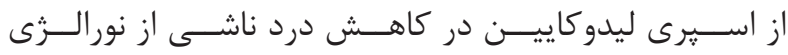

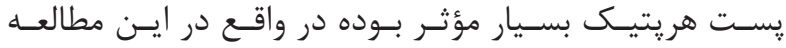

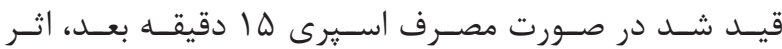

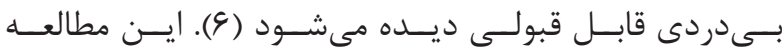

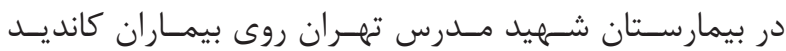

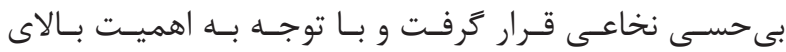

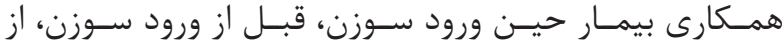

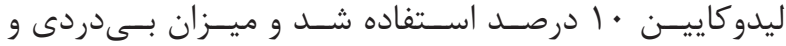

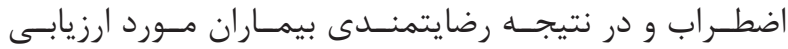

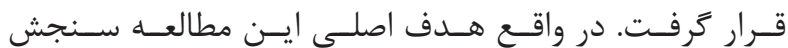

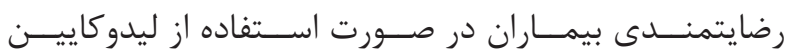

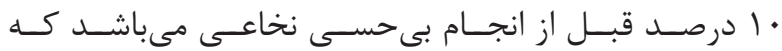

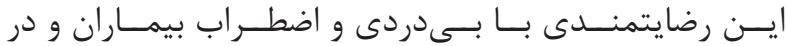

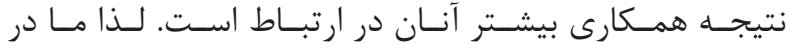

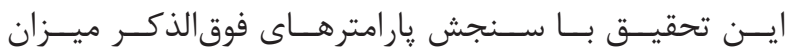

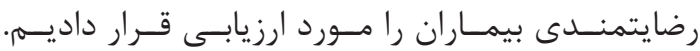

مواد و روشها

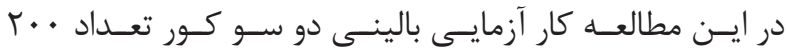

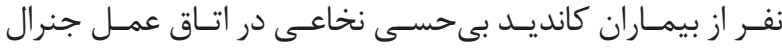

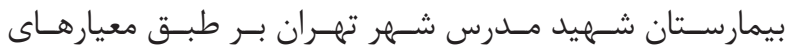

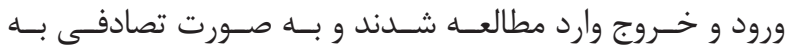

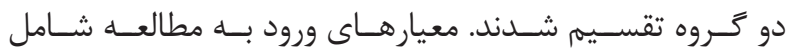

\footnotetext{
${ }^{1}$ Procedure

${ }^{2}$ Spinal anesthesia

${ }^{3}$ Intravenous cannulation

${ }^{4}$ Epidural anesthesia

${ }^{5}$ Local anesthetic gel

${ }^{6}$ Skin patch
}

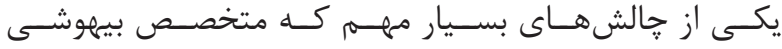

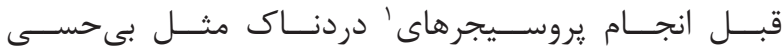

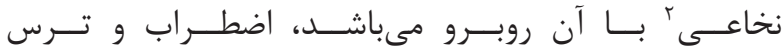

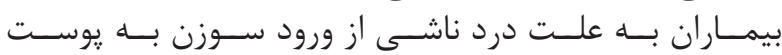

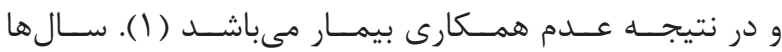

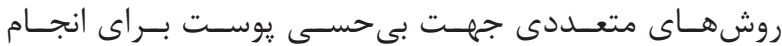

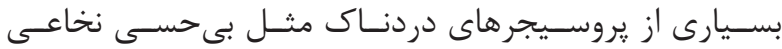

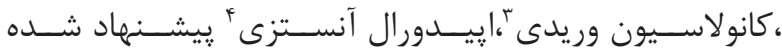

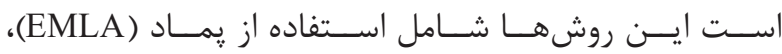

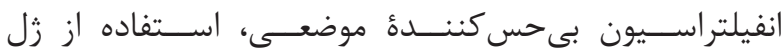

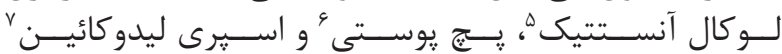

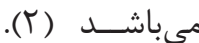

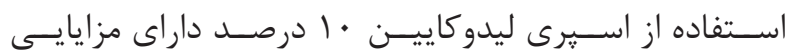

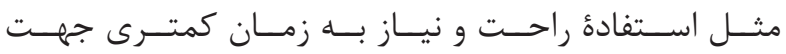

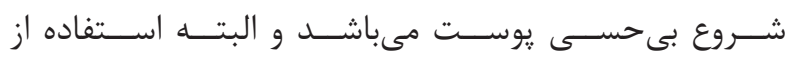

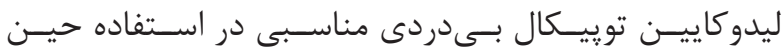

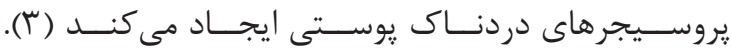

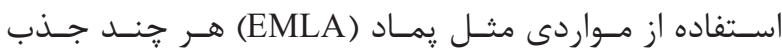

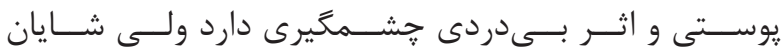

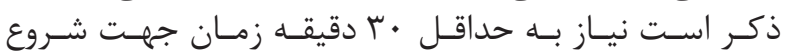

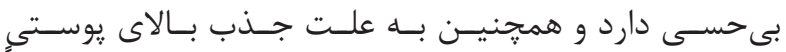

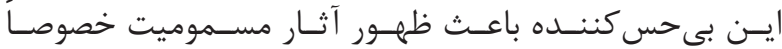

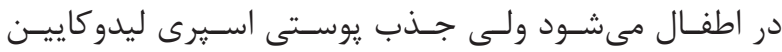

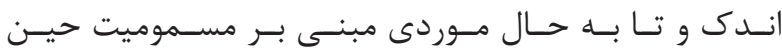

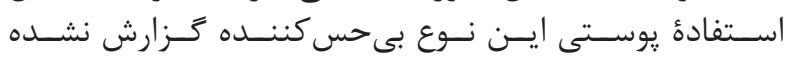

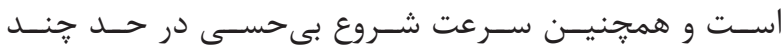

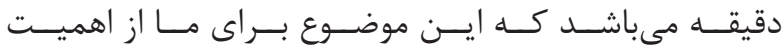

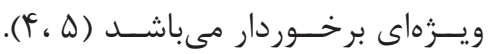

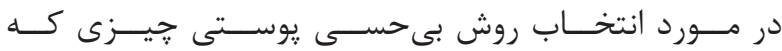

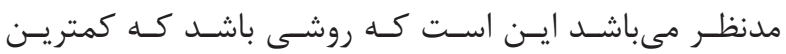

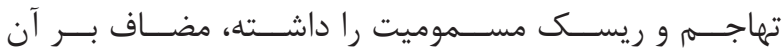

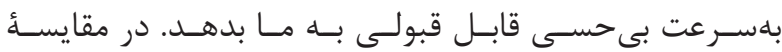

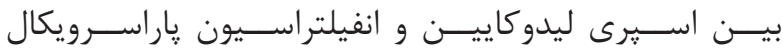

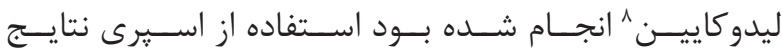

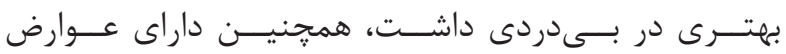

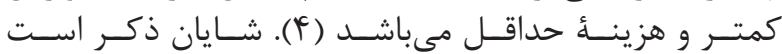

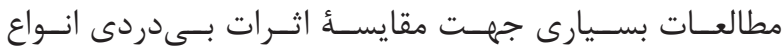

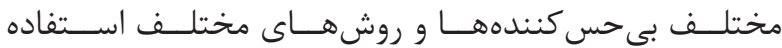

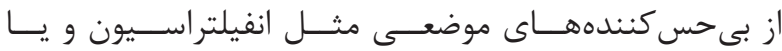

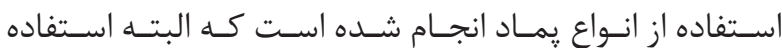

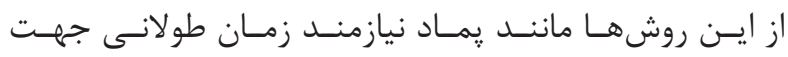

${ }^{7}$ Lidocaine spray

${ }^{8}$ Paracervical lidocaine infiltration

${ }^{9}$ Histosalpingography

${ }^{10}$ Pain score

${ }^{11}$ Visual analogue scale 


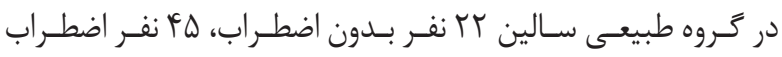

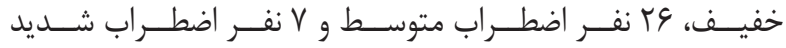

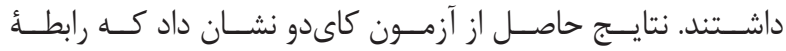

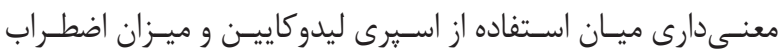

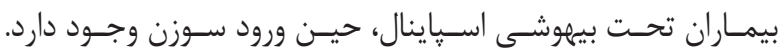
.(chi square $=\mathrm{V} / / \cdot \wedge \cdot)(P<\cdot / \cdot \cdot 1)$

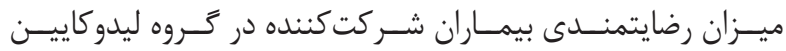

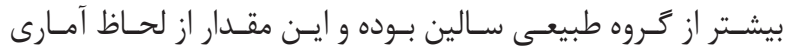

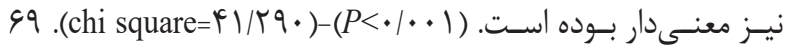

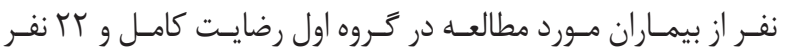

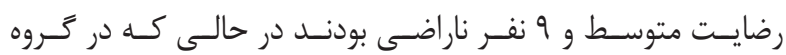

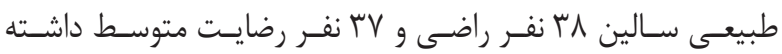

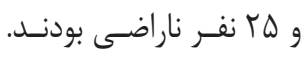

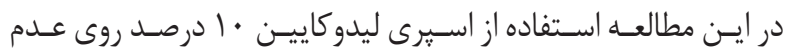

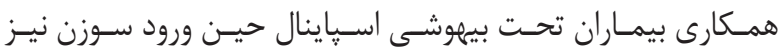

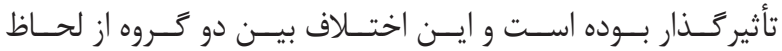

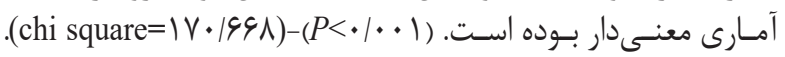

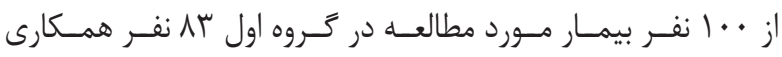

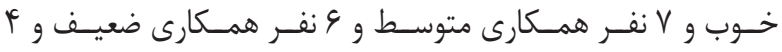

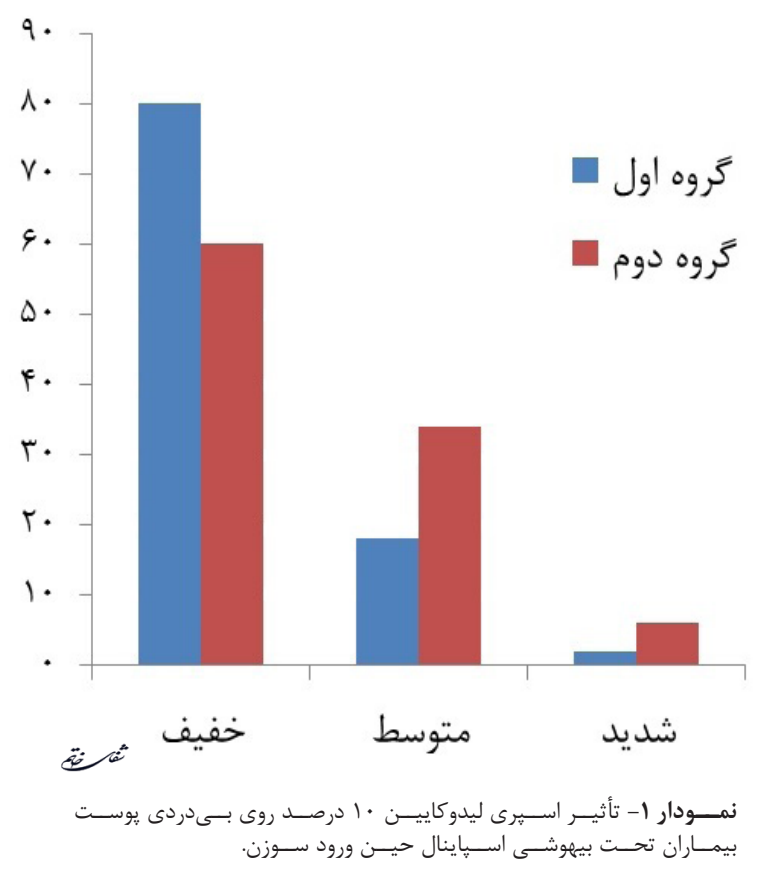

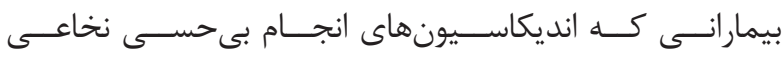

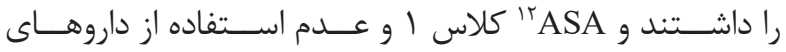

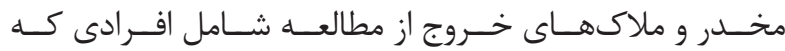

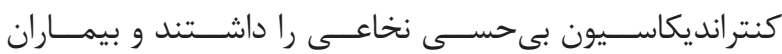

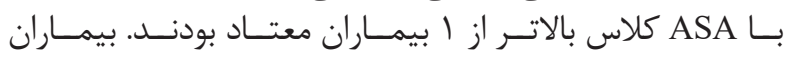

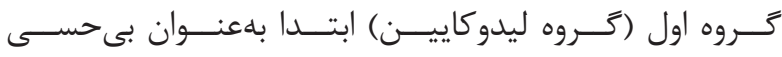

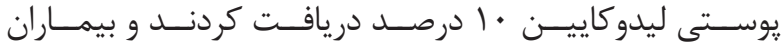

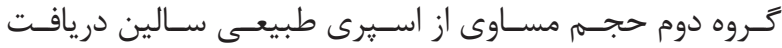

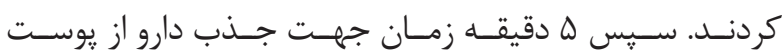

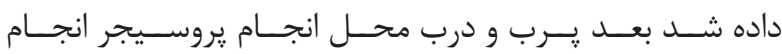

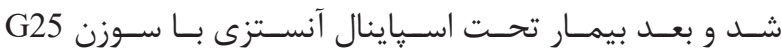

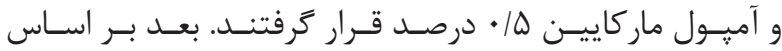

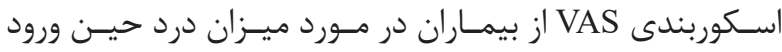

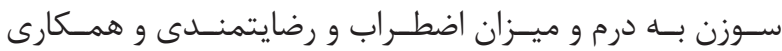

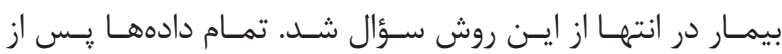

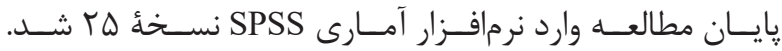

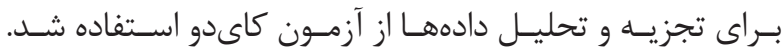

يافتهها

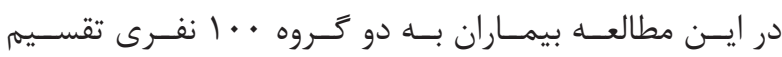

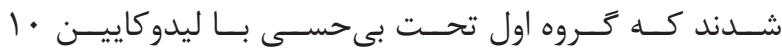

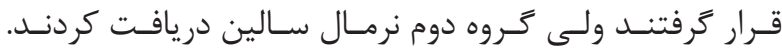

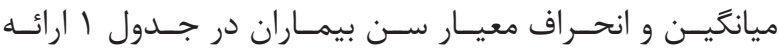
شـده اسـتـ

همانطـور كـهـ در جـدول ا نشـان داده شـــ ميانكَيـن سـن بيمـاران

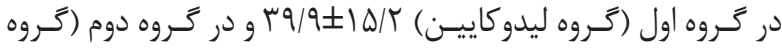

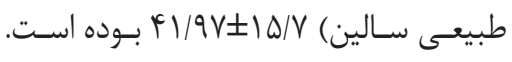

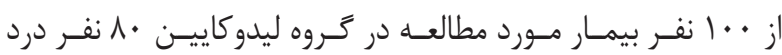

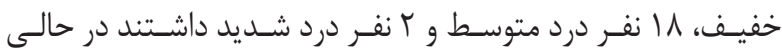

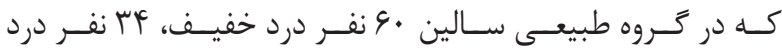

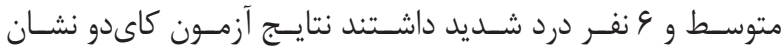

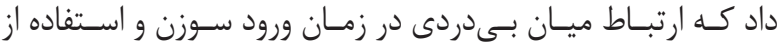

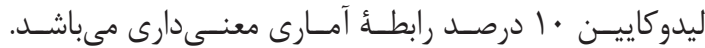
(chi square= $1 r \Delta / \Delta r \cdot)-(P<\cdot / \cdot \cdot 1)$

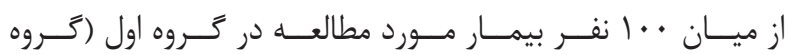

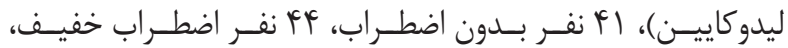

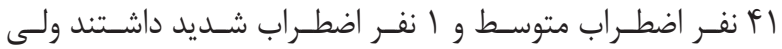

\begin{tabular}{|c|c|c|}
\hline nروه دوم . . & & متغيبير سـن \\
\hline$F / / 1 \Delta \pm 9 \vee / V$ & rq// & ميانَين سن \\
\hline 认 & 人 人 & كمترين سن \\
\hline Vq & Vq & بيشترين سـن \\
\hline
\end{tabular}

${ }^{12}$ American national standards institute 


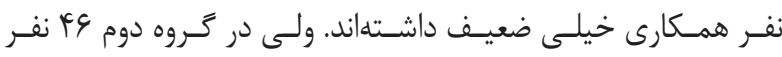

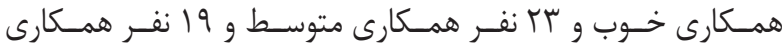

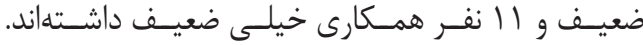

بحث و نتيجه

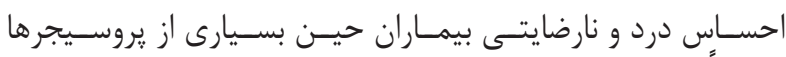

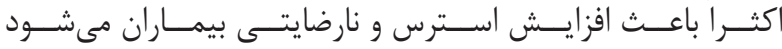

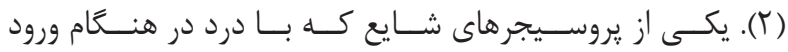

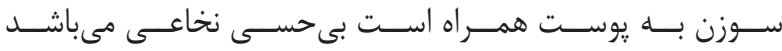

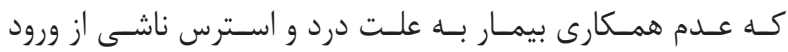

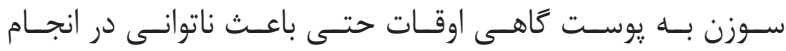

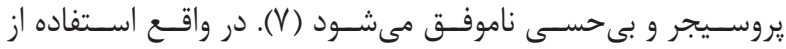

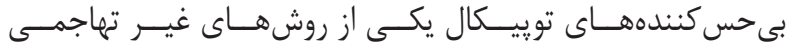

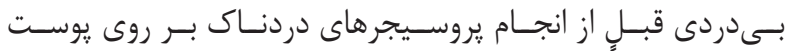

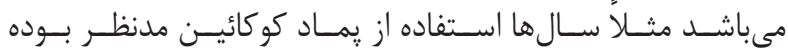

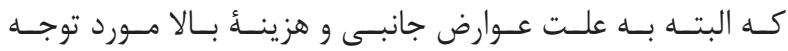

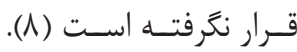

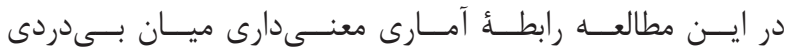

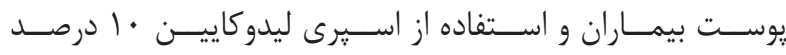

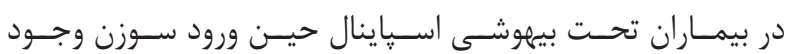

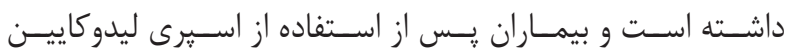

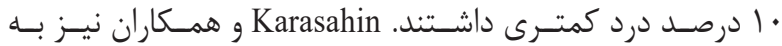

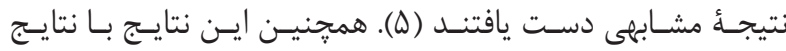

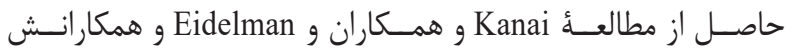

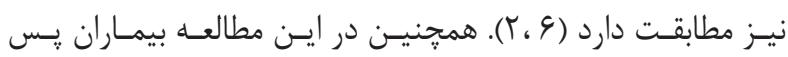

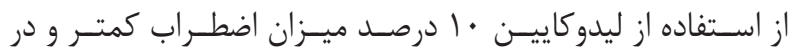

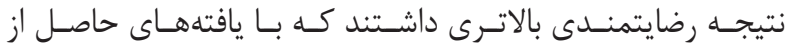

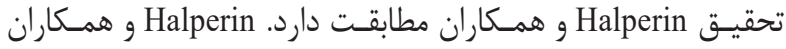

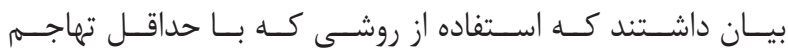

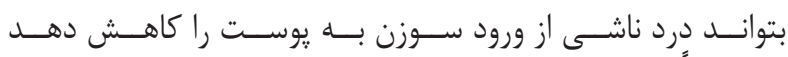

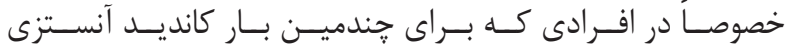

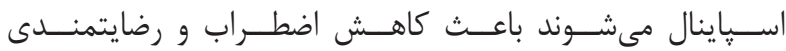

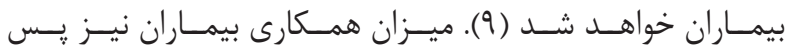

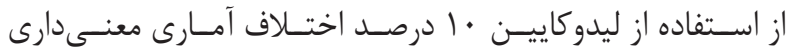

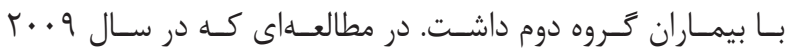

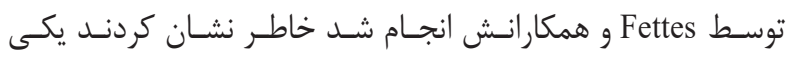

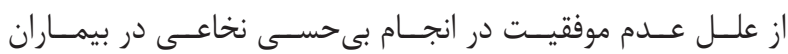

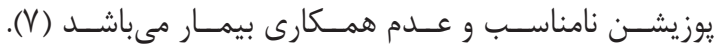

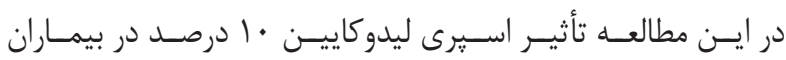

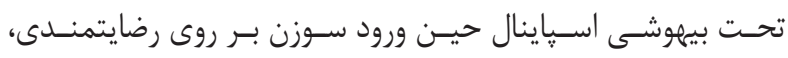

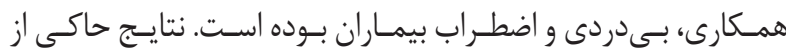

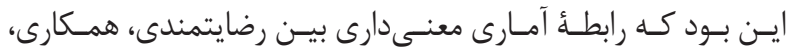

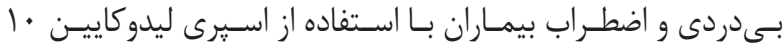

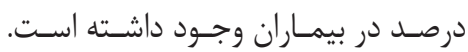

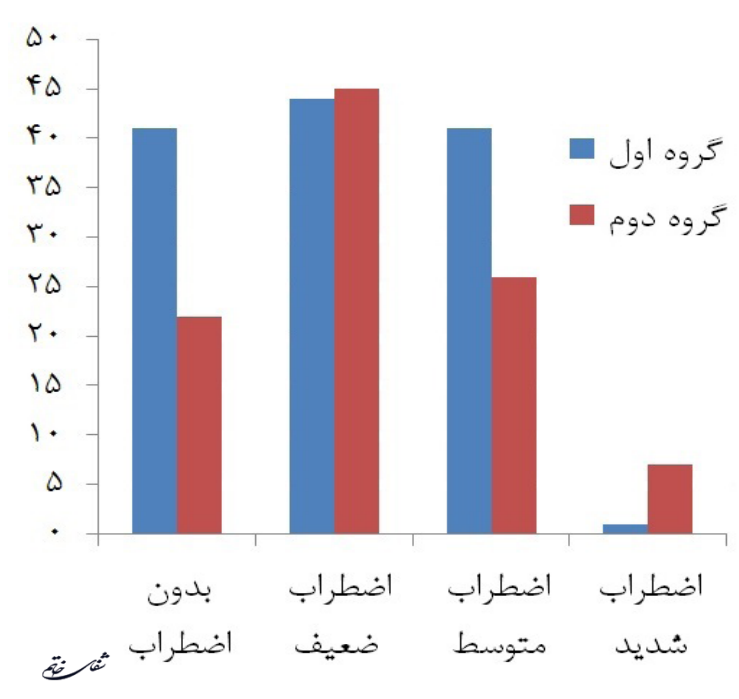

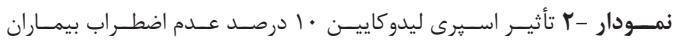

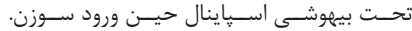

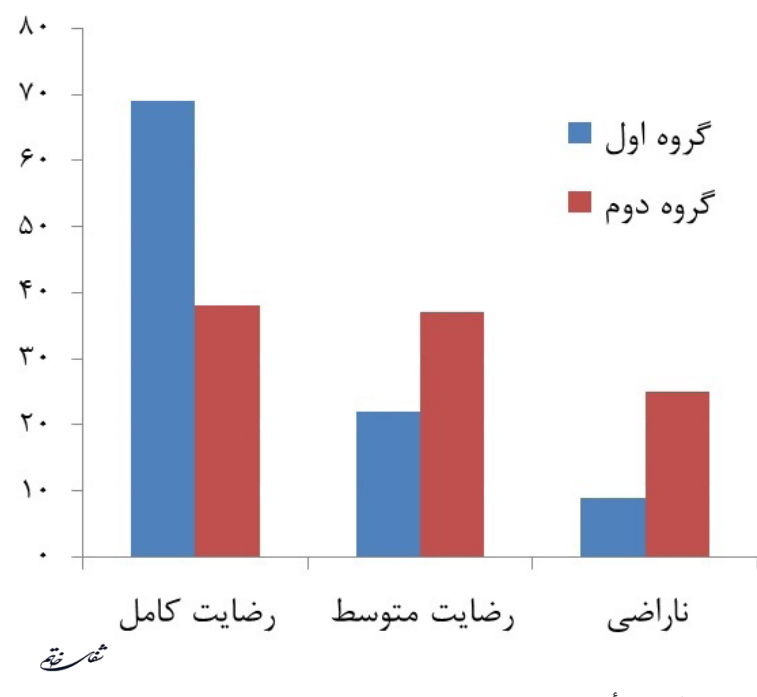

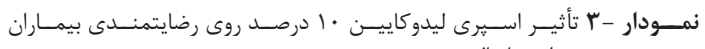

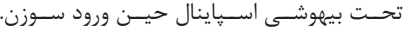

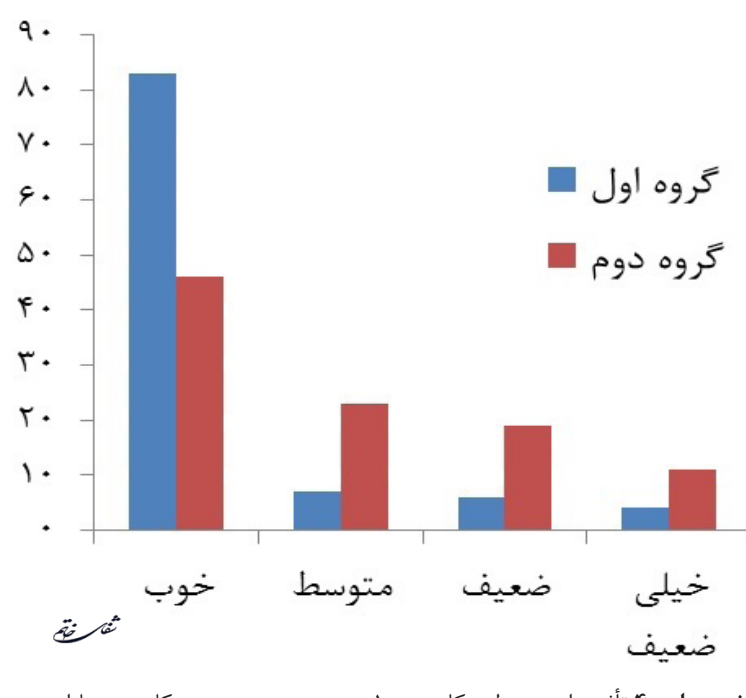

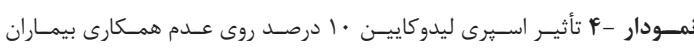
تحست بيهوشـى اسـياينال حيـن ورود سـوزن. 
1. Lepage C, Drolet P, Girard M, Grenier Y, DeGagné R. Music decreases sedative requirements during spinal anesthesia. Anesth Analg. 2001; 93(4): 912-6.

2. Eidelman A, Weiss JM, Lau J, Carr DB. Topical anesthetics for dermal instrumentation: a systematic review of randomized, controlled trials. Ann Emerg Med. 2005; 46(4): 343-51.

3. Mudge D, Younger JB. The effects of topical lidocaine on infant response to circulation. Journal of NurseMidwifery. 1989; 34(6): 335-40.

4. Castillo JG. Topical anesthetic formulation. Google Patents. 1999.

5. Soriano D, Ajaj S, Chuong T, Deval B, Fauconnier A, Darai E. Lidocaine spray and outpatient hysteroscopy: randomized placebo-controlled trial. Obstet Gynecol. 2000; 96(5): 661-4.

6. Selby I, Bowles B. Analgesia for venous cannulation: a comparison of EMLA (5 minutes application), lignocaine, ethyl chloride, and nothing. J R Soc Med. 1995; 88(5): 264-7.

\section{منابع}

7. Karasahin E, Alanbay I, Keskin U, Gezginc K, Baser I. Lidocaine $10 \%$ spray reduces pain during hysterosalpingography: a randomized controlled trial. J Obstet Gynaecol Res. 2009; 35(2): 354-8.

8. Kanai A, Kumaki C, Niki Y, Suzuki A, Tazawa T, Okamoto H. Efficacy of a metered-dose $8 \%$ lidocaine pump spray for patients with pos t-herpetic neuralgia. Pain Med. 2009; 10(5): 902-9.

9. Fettes P, Jansson J-R, Wildsmith J. Failed spinal anaesthesia: mechanisms, management, and prevention. $\mathrm{Br} \mathrm{J}$ Anaesth. 2009; 102(6): 739-48.

10. Eidelman A, Weiss JM, Enu IK, Lau J, Carr DB. Comparative efficacy and costs of various topical anesthetics for repair of dermal lacerations: a systematic review of randomized, controlled trials. J Clin Anesth. 2005; 17(2): 106-16.

11. Halperin DL, Koren G, Attias D, Pellegrini E, Greenberg ML, Wyss M. Topical skin anes thesia for venous, subcutaneous drug reservoir and lumbar punctures in children. Pediatrics. 1989; 84(2): 281-4. 